\title{
Bedform dynamics from coupled bed-flow direct numerical simulations
}

\author{
N. Zgheib ${ }^{1,2, *}$, J.J. Fedele ${ }^{3}$, D.C.J.D. Hoyal ${ }^{3}$, M.M. Perillo ${ }^{3}$, and S. Balachandar ${ }^{2}$ \\ ${ }^{1}$ School of Engineering, Lebanese American University, Byblos, Lebanon \\ ${ }^{2}$ Department of Mechanical and Aerospace Engineering, University of Florida, Gainesville, FL, USA \\ ${ }^{3}$ ExxonMobil Upstream Research Company, Houston, TX, USA
}

\begin{abstract}
We present results of time-evolving coupled direct numerical simulations between an erodible bed and an overlying pressure-driven, turbulent flow field. A total of 6 simulations are considered, the details of which are shown in Table 1 . The numerical setup consists of a horizontally periodic open channel, and the simulations are run at a shear Reynolds number of $R e_{\tau}=180$. The coupling between the spatially and temporally evolving sediment bed and the flow field is enforced through the explicit immersed boundary method (IBM) of Uhlmann [1]. The flow field is fully resolved and is obtained by integrating the conservation of mass and momentum equations using a pseudo spectral code [2]. On the other hand, the sediment bed is modelled via the Exner equation [3]. Details about the numerical approach are available in [4-5].
\end{abstract}

\section{Background}

Ripples are examples of bedform patterns that result from the instability of an erodible bed of particles subjected to the shearing action of an overlying flow field [6]. The evolution of ripples has been well documented experimentally and numerically. Here we show using coupled, flow-bed simulations that we are able to capture the various stages of ripple formation observed in experimental flumes.

\section{Results from direct numerical simulations}

Fig. 1 shows a top view and an isometric view with iso-contours of the bed-normal tangential velocity gradient. These simulations evolve from an initially flat bed. At the start of the simulation, the flow corresponds to a fully developed, $R e_{\tau}=180$ turbulent open channel flow over a flat bed. Shortly afterwards, longitudinal streaks are easily discerned (panel b). These are aligned predominantly with the flow direction with only a few aligned at an angle of about $45^{\circ}$. The Chevron features are next to emerge in panel c followed by the incipient crestlines in panel $d$. The incipient crestlines then coarsen and grow in amplitude to form straight and sinuous ripples (panels e and f). These ripples will continue

\footnotetext{
*Corresponding author: nadim.zgheib@1au.edu.lb
} 
to grow and develop (and are hence termed developing ripples) until they reach an equilibrium or fully developed stage.

Table 1. List of simulations. Particle and fluid densities are held constant at 1.57 and $1.015 \mathrm{~g} / \mathrm{cm}^{3}$, respectively. $\mathrm{Re}_{\mathrm{p}}$ is the particle Reynolds number. $\Theta_{\mathrm{cr}}$ is the critical Shields number and $\Theta$ is the

Shields number imposed by the flow. $\mathrm{u}_{\tau}$ and $\mathrm{H}_{\mathrm{f}}$ are the bed shear velocity and flow depth.

\begin{tabular}{|c|c|c|c|c|c|c|}
\hline $\begin{array}{c}\text { Simulation } \\
\text { number }\end{array}$ & $\begin{array}{c}\text { Particle diameter } \\
(\boldsymbol{\mu m})\end{array}$ & $\boldsymbol{R} \boldsymbol{e}_{\boldsymbol{p}}$ & $\boldsymbol{\Theta}_{\boldsymbol{c r}}$ & $\boldsymbol{\Theta}$ & $\begin{array}{c}\boldsymbol{u}_{\boldsymbol{\tau}} \\
(\mathbf{c m} / \mathbf{s})\end{array}$ & $\begin{array}{c}\boldsymbol{H}_{\boldsymbol{f}} \\
(\mathbf{c m})\end{array}$ \\
\hline S1 & 250 & 9.347 & 0.029 & 0.109 & 1.237 & 1.455 \\
\hline S2 & 150 & 4.344 & 0.046 & 0.182 & 1.237 & 1.455 \\
\hline S3 & 50 & 0.836 & 0.122 & 0.547 & 1.237 & 1.455 \\
\hline S4 & 250 & 9.347 & 0.029 & 0.055 & 0.874 & 2.058 \\
\hline S5 & 150 & 4.344 & 0.046 & 0.091 & 0.874 & 2.058 \\
\hline S6 & 50 & 0.836 & 0.122 & 0.274 & 0.874 & 2.058 \\
\hline
\end{tabular}

There is a rich spectrum of bedform interactions that occur during the development of the bed. [7] provide a thorough summary of the various bedform interactions documented in laboratory experiments as well as field studies. Some of these interactions may be constructive such as lateral linking and merging, which result in fewer, larger, and more widely spaced bedforms, or regenerative such as bedform splitting and defect creation, which push the system in the opposite direction towards a more preliminary state. Other interactions such as bedform and defect repulsion do not fall under the above two categories, but rather lead to bedform rearrangement through a pattern change.
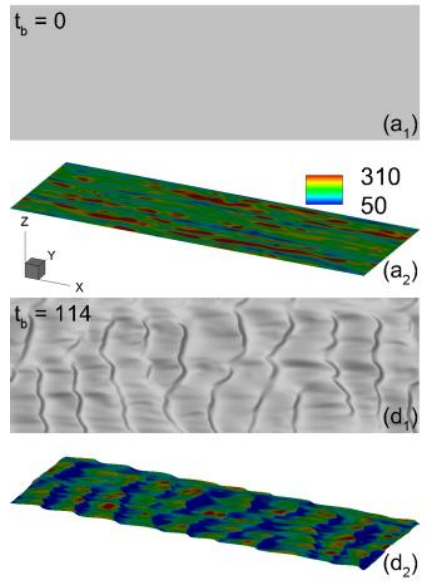

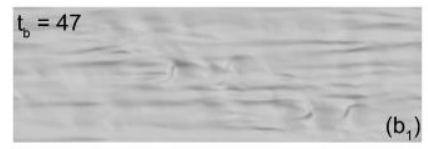

$\left(b_{1}\right)$
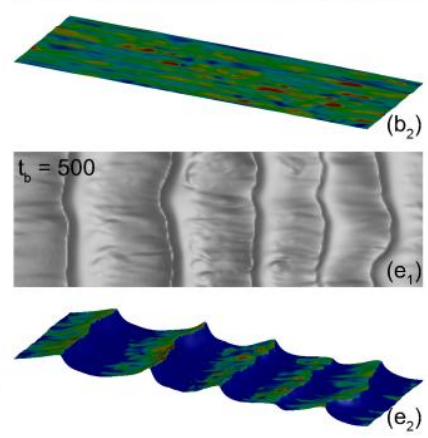
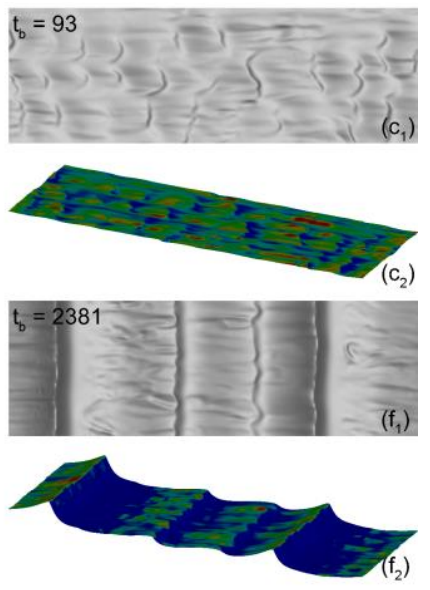

Fig. 1. Top (grayscale) and isometric (colored) views of the temporal evolution of the bed from S2 at six time instances. For each snapshot, the bed is shown in two different views. Flow is from left to right. The contours in the isometric view correspond to the bed-normal tangential velocity gradient.

In our simulations, we observe many such documented interactions including lateral linking, defect and bedform repulsion, defect creation, as well as merging. These interactions are present in all our simulations, but in the interest of brevity, we will show a single example of some of these interactions. In Fig. 2, we show a top view of the bed in which, for the purpose of clarity, bedform features are exaggerated by stretching the 
vertical direction by a factor of 4 . Except for frames $a_{1}$ and $a_{2}$, we show iso-contours of the sediment bed height. Frames $\mathrm{a}_{1}-\mathrm{a}_{3}$ show the bedform interaction of lateral linking.

In frames $b$, c, and $d$, we observe various flow-aligned bedform interactions known as defect repulsion, bedform repulsion, and merging, respectively. The mechanisms behind all these bedform interactions are discussed in detail in [4].
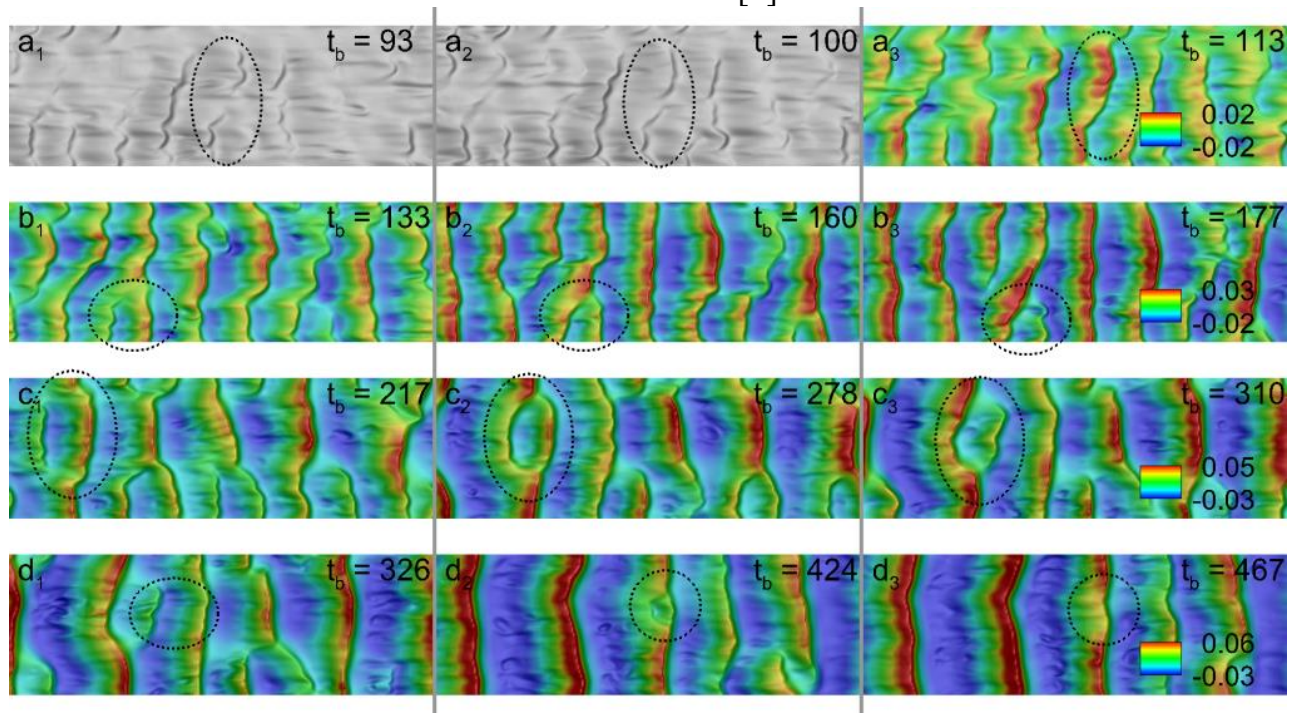

Fig. 2. Bedform interactions. See text for detail. The grayscale in the a panels is used for clarity.

\section{References}

1. Uhlmann, M. (2005). An immersed boundary method with direct forcing for the simulation of particulate flows. Journal of Computational Physics, 209(2), 448-476

2. Cantero, M. I., Balachandar, S., \& Garcia, M. H. (2007). High-resolution simulations of cylindrical density currents. Journal of Fluid Mechanics, 590, 437-469

3. Cayocca, F. (2001). Long-term morphological modeling of a tidal inlet: the Arcachon Basin, France. Coastal Engineering, 42(2), 115-142

4. Zgheib, N., Fedele, J. J., Hoyal, D. C. J. D., Perillo, M. M., \& Balachandar, S. (2018a). Direct numerical simulation of transverse ripples: 1. Pattern initiation and bedform interactions. Journal of Geophysical Research: Earth Surface.

5. Zgheib, N., Fedele, J. J., Hoyal, D. C. J. D., Perillo, M. M., \& Balachandar, S. (2018b). Direct Numerical Simulation of Transverse Ripples: 2. Self- Similarity, Bedform Coarsening, and Effect of Neighboring Structures. Journal of Geophysical Research: Earth Surface.

6. Kennedy, J. F. (1969). The formation of sediment ripples, dunes, and antidunes. Annual Review of Fluid Mechanics, 1(1), 147-168.

7. Kocurek, G., Ewing, R. C., \& Mohrig, D. (2010). How do bedform patterns arise? New views on the role of bedform interactions within a set of boundary conditions. Earth Surface Processes and Landforms, 35(1), 51-63. 